\title{
Pneumothorax after pacemaker implantation localized contralaterally to the side of implantation - a rare but possible complication
} \author{
Marcin Grabowski²,A-F \\ A - Research concept and design, B - Collection and/or assembly of data, C - Data analysis and interpretation, \\ D - Writing the article, E - Critical revision of the article, F - Final approval of article \\ 1. Medical University of Warsaw \\ 2. First Department of Cardiology, Medical University of Warsaw
}

Michał Kowara ${ }^{1, \mathrm{~A}-\mathrm{C}, \mathrm{E}}$, Jakub Zawadzki' ${ }^{2, \mathrm{D}}$, Marcin Michalak²,B-C, Agnieszka Kołodzińska2,B-C, Grzegorz Opolski²,E-F,

Address for correspondence:

Michał Kowara, Medical University of Warsaw

email: michal.kowara@wum.edu.pl

Jakub Zawadzki, First Department of Cardiology, Medical University of Warsaw email: zawadzkijakub@o2.pl

Marcin Michalak, First Department of Cardiology, Medical University of Warsaw email: mmajkelak@gmail.com

\begin{abstract}
Agnieszka Kołodzińska, First Department of Cardiology, Medical University of Warsaw email: aa.kolodzinska@wp.pl
\end{abstract}

Grzegorz Opolski, First Department of Cardiology, Medical University of Warsaw email: grzegorz.opolski@wum.edu.pl

Marcin Grabowski, First Department of Cardiology, Medical University of Warsaw email: grabowski.marcin@me.com

Received: 16.11 .2017

Revised: 23.12 .2017

Accepted: 28.12.2017

Final review: 27.12.2017

DOI: $10.24255 / \mathrm{hbj} / 81611$

\section{Key words:}

pneumothorax, pacemaker, implantation, subclavian vein puncture

\section{Abstract}

Case report - An 86-year old patient developed pneumothorax a few hours after pacemaker implantation. Surprisingly, the pneumothorax localized contralaterally to the site of subclavian vein puncture. It was treated with chest tube puncture,

\section{Case}

An 86-year-old male patient underwent dual chamber pacemaker implantation due to symptomatic second degree atrioventricular block 2:1. His medical history was also positive for paroxysmal atrial fibrillation, hypertension, and chronic obstructive pulmonary disease. No significant deviations were reported in laboratory tests.

The device (Medtronic Sensia DR) was implanted in the left prepectoral region via a subclavian approach. An active fixation ventricular lead (Medtronic 5076-58 MRI) was implanted into the right ventricular outflow tract through left under the care of a pneumonologist, resulting in full recovery. Although pneumothorax localized contralaterally to the site of subclavian vein puncture during pacemaker implantation is very rare, it has been reported in the literature.

cephalic vein venesection, while another active fixation atrial lead (Medtronic 4076-52) was located within the right atrial appendage using left subclavian vein puncture. The procedure went without perioperative complications. Post-procedure chest radiographs taken directly after the intervention were satisfactory.

Five hours later, the patient suddenly reported shortness of breath and a stabbing pain in the chest intensified by inspiration. A chest X-ray (posteroanterior view) revealed a right-sided (contralateral) pneumothorax (Figure 1), while the lateral view appeared normal (Figure 2). A chest tube 


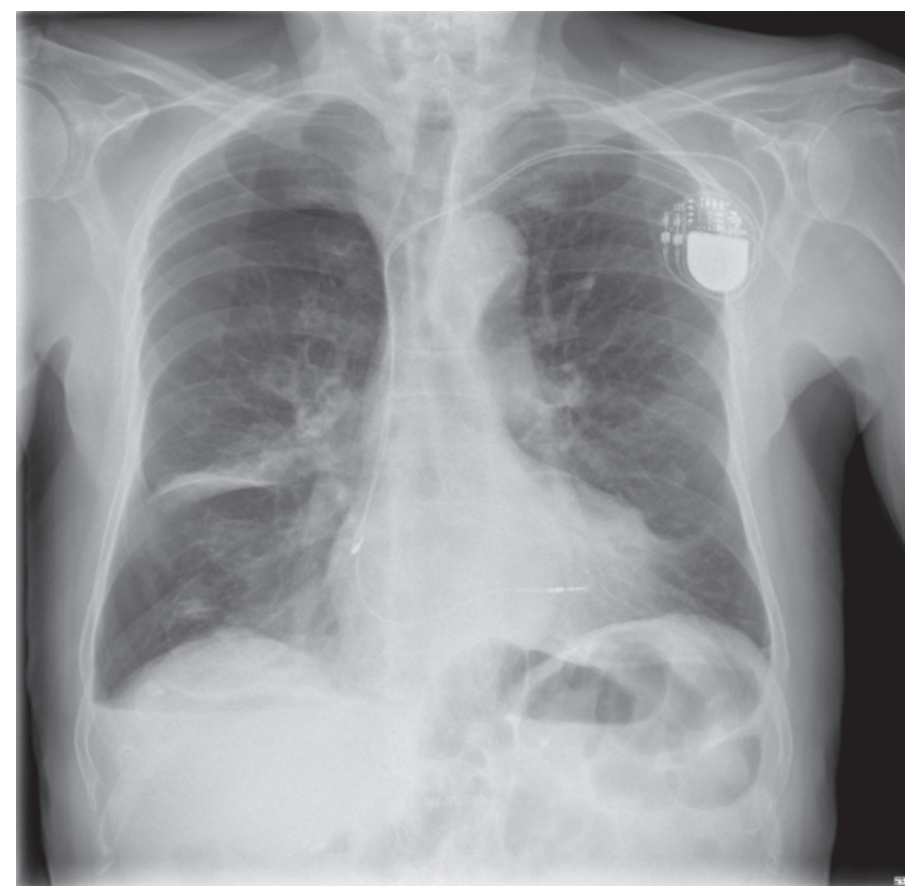

Figure 1. Chest $\mathrm{x}$-ray in the posteroanterior view revealing right-sided pneumothorax.

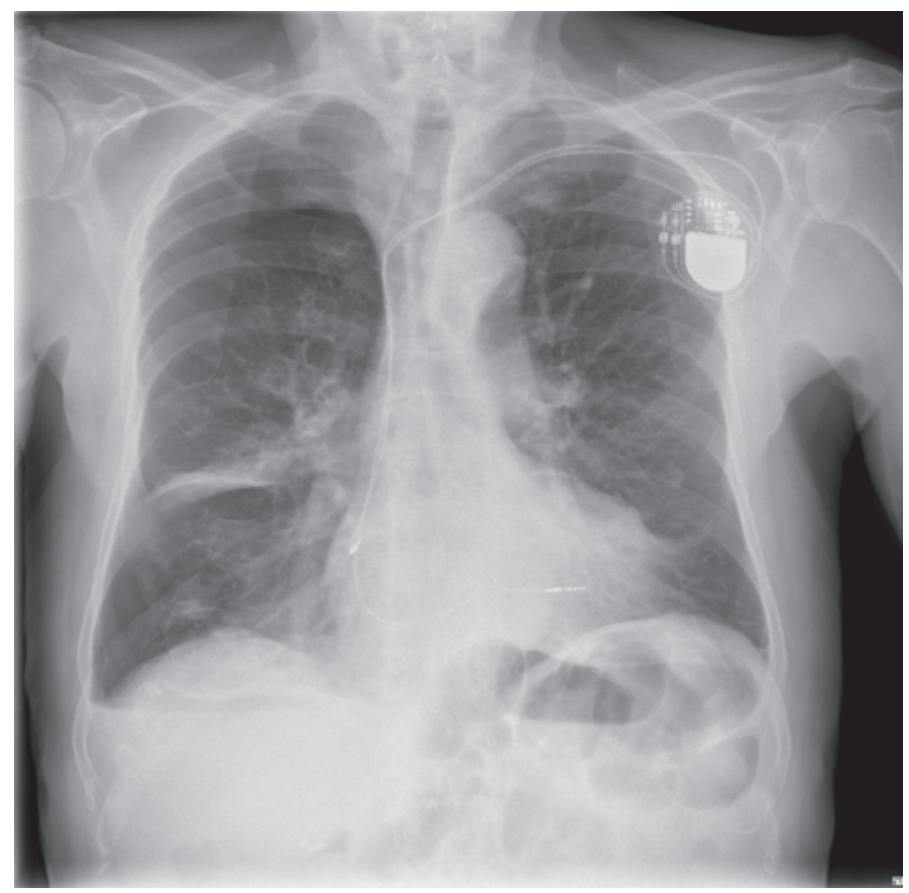

Figure 2. Position of leads appears normal in the posteroanterior view.

was inserted in the third intercostal space, evacuating 720 $\mathrm{ml}$ of air and promptly relieving the symptoms. Pacemaker interrogation revealed no change in lead data or pacemaker activity parameters.

During several days of in-hospital observation the patient was found to be in a good general condition, leads' electrical parameters were stable, and function of the pacing system was proper.

\section{Discussion}

The patient was admitted to our department with second-degree type 2 atrioventricular block presenting a class I recommendation for pacing, irrespective of symptoms, and there is evidence of superiority of dual-chamber pacing over only ventricular pacing ${ }^{[1]}$.

Pneumothorax as an early complication occurs with a frequency between $0.9 \%$ and $1.9 \%$ and next to lead dislodgement/ loss of capture is one of the most common complications ${ }^{[2,3]}$. However, our patient's pneumothorax occurred on the opposite side of the procedure. This situation is possible, although rarely described in publications ${ }^{[4-9]}$. The first case of right-sided pneumothorax contralateral to the site of implantation was described in 1999 and occurred in a 79-year-old, small-built Taiwanese woman (body weight of $51 \mathrm{~kg}$ and height of $140 \mathrm{~cm})^{[10]}$.

Presumably this situation can be caused by incidental subclavian or brachiocephalic vein puncture during lead insertion and subsequent puncture of the right pleura (as described $\left.\mathrm{in}^{[8]}\right)$. Another situation in which contralateral right-sided pneumothorax can occur is atrial lead extrusion through the right atrial appendage, which abuts the right pleura [7]. Last but not least, atrial wall perforation caused directly by the atrial lead can also cause right-sided pneumothorax with or without pericardial effusion ${ }^{[6,11]}$. Importantly, atrial leads are implanted by an active fixation (screw-in) mechanism to the atrial wall, which increases the risk of perforation. Therefore these leads must be implanted with caution and overscrewing should be avoided ${ }^{[10]}$. In our case no intra-procedural problems with leads' insertion or implantation were reported by the operator. Overscrewing of the atrial lead is rather unlikely because of the specified number of clockwise turns that are done during lead fixation under fluoroscopic control. However, our patient had a history of longstanding smoking and chronic obstructive pulmonary disease and received chronic therapy of inhaled fluticasone propionate and salmeterol xinafoate. This situation increases the risk of complications, given that a case of contralateral pneumothorax in a patient on long-term steroid therapy has been described ${ }^{[12]}$.

In conclusion, the case of our patient shows that contralateral right-sided pneumothorax as a complication of pacemaker implantation is possible, even though very rare. Moreover, a patient can manifest the symptoms many hours after the operation. In our case it was 5 hours, in the first case described in the literature it was 4 hours ${ }^{[10]}$, but in the case described by Dilling-Boer D and colleagues it was 3 days $^{[6]}$. Therefore patients discharged from hospital shortly after the intervention should remain in direct contact with the electrotherapy center, which enables an appropriate response as soon as possible when necessary.

\section{References}

1. Brignole M, Auricchio A, Baron-Esquivias G, et al. 2013 ESC Guidelines on cardiac pacing and cardiac resynchronization therapy: The Task Force on cardiac pacing and resynchronization therapy of the European Society of Cardiology (ESC). Developed in collabora- 
tion with the European Heart Rhythm Association (EHRA). European Heart Journal (2013) 34, 2281-2329

2. Armaganijan LV, Toff WD, Nielsen JC et al. Are elderly patients at increased risk of complications following pacemaker implantation? A meta-analysis of randomized trials. Pacing Clin Electrophysiol. 2012 Feb;35(2):131-4.

3. Res JC, de Priester JA, van Lier AA et al. Pneumothorax resulting from subclavian puncture: a complication of permanent pacemaker lead implantation. Neth Heart J. 2004 Mar;12(3):101-105.

4. Pettemerides V, Jenkins N. Contralateral pneumothorax following repositioning of an atrial lead. Europace. 2012 Apr;14(4):606.

5. M Hardzina, A Ząbek, K Boczar et al. Contralateral pneumothorax after cardiac pacemaker implantation. Postepy Kardiol Interwencyjnej. 2015; 11(4): 347-348.

6. Dilling-Boer D, Ector H, Willems R et al. Pericardial effusion and right-sided pneumothorax resulting from an atrial active-fixation lead. Europace. 2003 Oct;5(4):419-23.

7. Srivathsan K, Byrne RA, Appleton CP et al. Pneumopericardium and pneumothorax contralateral to venous access site after permanent pacemaker implantation. Europace. 2003 Oct;5(4):361-3.

8. Yada H, Sato T, Soejima K et al. Images in cardiovascular medicine. Pseudonormal position of an atrial pacemaker lead associated with a contralateral pneumothorax: where is the atrial lead? Circulation. 2008 Apr 29;117(17):2297-8.

9. Van Herendael H, Willems R. Contralateral pneumothorax after endocardial dual-chamber pacemaker implantation resulting from atrial lead perforation. Acta Cardiol. 2009 Apr;64(2):271-3.

10. Ho WJ, Kuo CT, Lin KH. Right pneumothorax resulting from an endocardial screw-in atrial lead. Chest. 1999 Oct;116(4):1133-4.

11. Oginosawa $Y$, Abe H, Nakashima Y. Right pneumothorax resulting from an endocardial screw-in atrial lead in an implantable cardioverter defibrillator system. Pacing Clin Electrophysiol. 2002 Aug;25(8):1278-9.

12. Ishizue N, Kishihara J1, Niwano S1, Ako J1. Subacute pneumothorax contralateral to the venous access site associated with atrial lead perforation in a patient who was receiving corticosteroid therapy. J Arrhythm. 2017 Aug;33(4):335-337. 